\title{
Blind Identification of Space-time Block Coding (STBC) Using Single Receive Antenna over Frequency-selective Fading Channels
}

\author{
Hong-Wen Lin, Ke-Yuan Yu, Zhao-Gen Zhong \\ Department of Electronic and Information Engineering, Naval \\ Aeronautical and Astronautical University, \\ Yan Tai 264001, China \\ E-mail: Lin_hongwen@163.com,gfsskfqp@163.com, \\ zhongzhaogen@163.com
}

\begin{abstract}
Blind recognition of $\mathrm{STBC}$ is an important issue in the non-cooperative context. However, the research on blind recognition over frequency-selective fading channels has been rarely reported. This paper introduces a novel and efficient algorithm for the frequency-selective fading channels to classify STBC via Kolmogorov-Smirnov (K-S) test when a single antenna is employed at the receiver. The proposed algorithm exploits the discrimination features provided by the empirical cumulative distributions (CDFs) of the received signal. The distance between CDFs is employed relies on the two-sample Kolmogorov-Smirnov test. The algorithm's performance is compared under the condition in different modulation mode, sampling factors and confidence interval. Monte Carlo simulations are conducted to demonstrate the well performance of the proposed algorithms, and the proposed algorithm does not need the estimation of channel, noise statistics and modulation type, and, consequently, is well-suited for non-cooperative communication context.
\end{abstract}

Keywords-blind identification of STBC; frequency-selective fading channels; Kolmogorov -Smirnov (K-S) test; single receive antenna

\section{INTRODUCTION}

Signal identification is the process of identifying the type of the transmitted signal from a pool of candidates. More recently, multi-input-multi-output technology (MIMO) system with its full advantage of space resources, has received growing attention in military and academia, which may become the next generation of wireless communication systems in key technologies. Space-time block code (STBC) as a MIMO-based system, its blind identification [1, 2, 3] becomes an essential part of transmission parameter estimation. STBC identification algorithms can be divided into two categories: the maximum likelihood (ML) [4, 5] and the feature based (FB) $[6,7]$. The former provides the correct recognition probability of the optimal solution, Vincent Choqueuse and MlanieMarazin proposed an algorithm based on second-order statistics (SOS).However, the algorithm needs to know the channel information in advance, such as the noise power and carrier frequency offset. With the FB method, features are extracted from the received signal, and a decision

\author{
Shuai Liu \\ Department of Control Engineering, Naval Aeronautical and \\ Astronautical University, Yan Tai 264001, China \\ E-mail: Lin_hongwen@163.com,gfsskfqp@163.com, \\ zhongzhaogen@163.com
}

is made based on the detected characteristic values to identify different STBC. Correlation matrix Frobenius Norm Delay was investigated as identification features of the algorithm proposed by Vincent Choqueuseto identify the different $\mathrm{STBC}$ in [7].

To the best of the authors' knowledge, most studies of STBC identification algorithms considered frequency-flat fading channels [3, 4, 8]. However, in the actual communication environment, the channel propagation conditions are more severe, so the radio channel characteristics change over time, seriously affecting the quality of communication. In order to investigate the usefulness of STBC in the practical wireless channel, it is necessary to identify STBC over frequency-selective fading channels.

Different from the existing work, in this paper we adopt a novel and efficient algorithm for the frequency-selective fading channels to classify STBC via Kolmogorov-Smirnov (K-S) test when a single antenna is employed at the receiver. The transmitted signals propagate through frequency-selective fading channels, then the signals are split into two non-overlapping sequences. The algorithm relies on the Kolmogorov-Smirnov (K-S) test, and employs the maximum distance between the empirical cumulative distribution functions of the two statistics to recognize spatial multiplexing space-time block code (SM-STBC) and Alamouti space-time block code (AL-STBC).

The rest of the paper is organized as follows: Section II provides the signal model and frequency-selective fading channels. Section III introduces the proposed identification algorithm. Simulation results are provided in Section IV. Finally, conclusions are drawn in Section V.

\section{SIGNAL MODEL AND FREQUENCY-SELECTIVE CHANNELS}

\section{A. Signal Model}

We consider a wireless communication system using either SM or AL-STBC. Let $N_{\mathrm{t}}$ denote the number of transmit antennas, $\mathrm{N}$ be the number of symbols to be 
transmitted in each code and $\mathrm{L}$ be the number of time periods. The encoder employs a transmission matrix $\boldsymbol{C}(\boldsymbol{S})$ of size $N t \times L$ to map the symbols to be transmitted.

It is defined as a group of code symbols to be transmitted, which are given respectively as $\boldsymbol{S}=\left[\boldsymbol{s}_{1}, \boldsymbol{s}_{2}, \ldots \boldsymbol{s}_{n}\right]$. It is assumed to pass through the same linear modulation signal modulated multiplexed signal. Without loss of generality, it is assumed to be the first received signal, denoted by $\mathrm{r}(0)$, corresponding to the $(\mathrm{k}+1)$ th column of the jth transmitted signal, expressed as $\boldsymbol{C}_{k_{1}}\left(\boldsymbol{S}_{j}\right)$. In the additive noise, the kth received symbol is given by:

$$
r(k)=\boldsymbol{H} \boldsymbol{X}(k)+w(k)
$$

where

$$
\boldsymbol{X}(k)=\boldsymbol{C}_{\mathrm{u}}\left(\boldsymbol{S}_{\mathrm{v}}\right)
$$

with $u=\left(k+k_{1}\right) \bmod L, v=j+\left(k+k_{1}\right) \operatorname{div} L$ and $\mathrm{z} \bmod \mathrm{L}$ represent the remainder and the quotient of the division, respectively, $w(k)$ is the additive white noise, and $\boldsymbol{H}=\left[h_{l}, \ldots, h_{n_{t}}\right]$ is the fading coefficients vector.

\section{B. Frequency-selective Fading Channels}

In general, the frequency selective channel delay spread is relatively large, so that the received symbols of the same time is determined by a number of different transmission symbol weighted summation. The frequency selective channel will bring inter-symbol interference (ISI), which must be modeled by inter-symbol interference. Suppose that the number of multipath exists is $\mathrm{P}$ in some point, the relationship of discrete input and output over frequency-selective channel can be denoted as:

$$
\boldsymbol{r}_{t}=\sum_{p=0}^{P-1} \theta^{p} s_{t-p}+\boldsymbol{w}
$$

Suppose channel meet the following criteria [10]: (1) fading over each channel independently (2) having a temporal symmetry (3) all multipath can be accurately separated over each channel. The received signal can be expressed as a convolution of transmission signal and multipath channel, and the channel is composed of the multipath Rayleigh channel and Gaussian white noise composition after receiving the signal undergoes frequency-selective fading channel, the kth received symbols may be expressed as:

$$
\boldsymbol{r}(k)=\sum_{i=0}^{N_{i}-1} \sum_{p=0}^{p a t h-1} h_{i}(p) X^{i}(k-p)+\boldsymbol{w}(k)
$$

Where $\boldsymbol{w}(k)$ is the additive white Gaussian noise (AWGN).

\section{STBC Selection}

SM-STBC and AL-STBC are mainly adopted in the identification, because these codes respects the most popular STBCs in wireless communications standards.

SM-STBC: transmitting antenna number $n_{t}=j$, the code matrix length $L=1$

$\boldsymbol{C}(\boldsymbol{S})=s_{j}, j=1,2,3 \ldots$,

AL-STBC: transmitting antenna number $n_{t}=2$, the length of code matrix $L=2$

$$
\boldsymbol{C}(\boldsymbol{S})=\left(\begin{array}{cc}
s_{1} & -s_{2}^{*} \\
s_{2} & s_{1}^{*}
\end{array}\right)
$$

\section{K-S BASED IDENTIFICATION ALGORITHM}

At the receive-side, the sequence is denoted as [8]

$$
\boldsymbol{y}=[r(0), r(1), \ldots r(K-1)]
$$

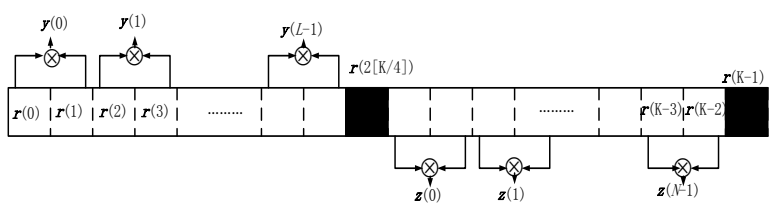

Figure 1. The method of signal sequence classification.

As shown in Figure 1, while the delay parameter $\tau=1$, the received signal is divided into two non-overlapping sequences

$$
\boldsymbol{y}_{1}=[r(0), r(1), \ldots r(2\lfloor K / 4\rfloor-1)]
$$

And

$$
\boldsymbol{y}_{2}=[r(2\lfloor K / 4\rfloor+1), r(2\lfloor K / 4\rfloor+2), \ldots, r(K-2)]
$$

$\mathrm{K}$ represents the number of received symbols.

Let us define the correlation function $Y(k)$ and $Z(k)$

$$
\begin{gathered}
Y(k) \triangleq r(2 k) r(2 k+1) \\
Z(k) \triangleq r(2 k+2\lfloor K / 4\rfloor+1) r(2 k+2\lfloor K / 4\rfloor+2)
\end{gathered}
$$

When SM is transmitted, the components of both $Y(k)$ and $Z(k)$ are independent and identically distributed (i.i.d.), on the contrary, for AL-STBC, the components of $Y(k)$ and $Z(k)$ are not necessary i.i.d.

As to the alignment of the STBC block is not known at the blind receiver, we assume that $\mathrm{AL}-\mathrm{STBC}$ is received, which may have two different cases as follows:

- Case 1: for which the first received symbol does not correspond to the beginning of the AL-STBC block, 
$Y(k)$ represents the multiplication of consecutive received symbols from different blocks, whereas $Z(k)$ from the same blocks. It is obviously that $Y(k)$ is not an i.i.d. sequence, whereas for $Z(k)$ is an i.i.d. sequence.

- Case 2: for which the first received symbol is correspond to the beginning of the AL-STBC .By following the same reasoning, we can got a conclusion that $Y(k)$ is an i.i.d. sequence and $Z(k)$ is not.

Different STBC corresponding correlation function shows different distributions, which can as a feature to distinguish SM and AL-STBC by testing the null hypothesis.

$H_{0}: Y(k)$ and $Z(k)$ are distributed independently and identically distributed.

$H_{1}: Y(k)$ and $Z(k)$ are not all independent and identically distributed, and do not obey the same distribution.

To measure the distance between two empirical CDFs, we employ the two-sample Kolmogorov-Smirnov (K-S) test [14]. Let $F_{y}(\mathrm{x})$ and $F_{z}(\mathrm{x})$ denote the empirical cumulative distributions (CDFs) of the sequences $Y(k)$ and $Z(k)$, which are defined as

$$
\begin{aligned}
& \hat{F}_{\mathrm{y}}(\mathrm{x}) \triangleq \frac{1}{L} \sum_{n=0}^{L-1} \operatorname{Ind}(\mathbf{Z}(\mathrm{n})<\mathrm{x}) \\
& \hat{F}_{\mathrm{Z}}(x) \triangleq \frac{1}{N} \sum_{\mathrm{n}=0}^{N-1} \operatorname{Ind}(\mathbf{Z}(\mathrm{n})<\mathrm{x})
\end{aligned}
$$

Where $\operatorname{Ind}($.$) denotes the indicator function. While the$ input parameter is true, the indicator function value is 1 , otherwise, the indicator function value is zero.

The maximum distance between two empirical cumulative distribution function can be expressed as

$$
T_{K-S} \triangleq \max _{v}\left|F_{z}(v)-F_{y}(\mathrm{v})\right|
$$

Where

$\mathrm{v} \in\{\mathrm{y}(0), \ldots \mathrm{y}(\mathrm{L}-1), \mathrm{z}(0), \ldots \mathrm{z}(\mathrm{N}-1)\} \quad$. Compared the goodness-of-fit statistic with a threshold, $\lambda$, the hypothesis $H_{0}$ is rejected, while

$$
T_{K-S} \geq \lambda
$$

And

$$
P\left(H=H_{o} \mid H_{o}\right)=P\left(T_{K-S}<\lambda \mid H_{o}\right)
$$

Where $H$ is the estimated $\mathrm{K}-\mathrm{S}$ test, the confidence interval $\beta$ can be expressed as

$$
\beta=1-\Phi\left(\lambda\left[\sqrt{\frac{N L}{N+L}}\right]+0.12+\frac{0.11}{\sqrt{\frac{N L}{N+L}}}\right)
$$

With

$$
\Phi(x) \triangleq 2 \sum_{i=1}^{\infty}(-1)^{i-1} e^{-2 i^{2} x^{2}}
$$

\section{SIMULATION RESULTS}

\section{A. Simulation Setup}

Monte Carlo simulations were carried out to investigate the performance of the proposed identification algorithm Each set of simulations was run for 1000 trials. Each experimental condition is frequency-selective Rayleigh fading channel, its PDF obey exponential model. Each channel tap is modeled as independent complex Gaussian random variable, its power obeys the exponential PDF, the maximum number of path is four. Unless otherwise mentioned, Quadrature phase shift keying (QPSK) was adopted Noise with zero mean additive white Gaussian noise , the number of received signal samples $\mathrm{K}=8192$, the confidence intervals $\beta=0.99$. Two recognition probabilities were adopted to measure the simulation result, one is the average probability of correct identification. $P_{c}=\frac{1}{2} \sum_{\theta \in\{\mathrm{SM}, \mathrm{AL}\}} P(\theta=\theta \mid \theta)$, the other is the probability of correct identification $P(\theta=\theta \mid \theta), \theta \in\{\mathrm{SM}, \mathrm{AL}\}$, with $\theta$ as the estimated signal type.

\section{B. Simulation Results}

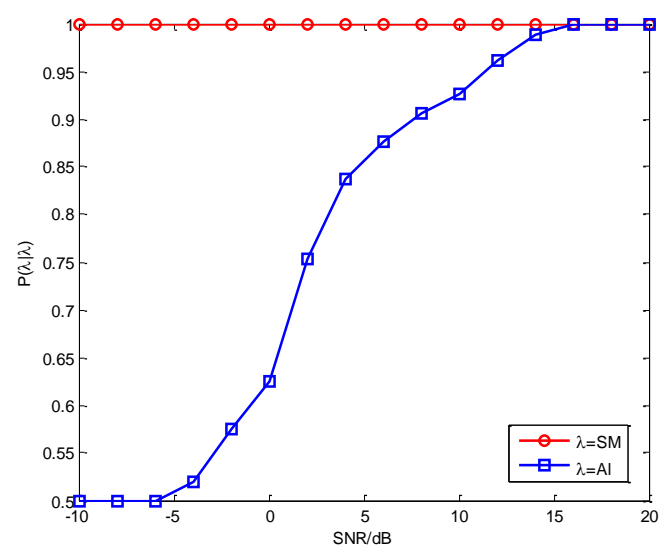

Figure 2. The probability of correct identification between different STBC. 


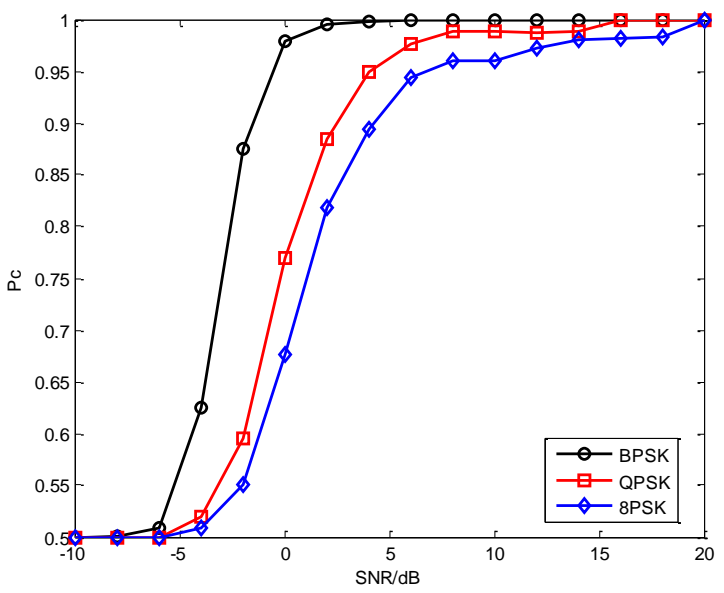

Figure 3. The analysis of modulation mode and recognition probability.

Figure 2 shows the probability of correct identification for SM-STBC and AL-STBC over frequency-selective fading channel. As shown, the probability of correct identification for $\mathrm{SM}$, is equal to the target confidence interval $\beta=0.99$, and is independent of the signal noise ratio (SNR). For AL-STBC, its probability of correct identification improves as the SNR increase. Due to the low SNR, as the noise reduced, the empirical cumulative distribution function becomes small. With the SNR increasing, the effects of noise are limit, so the performance of the identification algorithm behaves better in turn.

Figure 3 presents the average probability of correct identification as a function for different modulation type. Consider three kinds of modulation type: binary phase-shift-keying (BPSK), Quadrature phase-shift-keying (QPSK) and 8 phase shift keying (8PSK).BPSK modulation is a real signal, the proposed algorithm can gain better performance in real transmission signal, especially while SNR is approximately $2 \mathrm{~dB}$ to achieve better performance. Compared to 8PSK, QPSK has a slightly advantage, but the overall difference between the two is not significant.

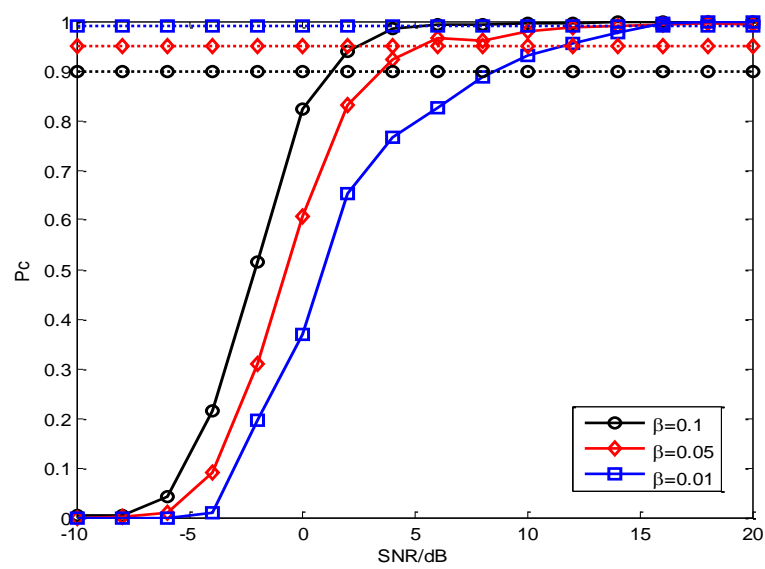

Figure 4. Correct recognition probability under different confidence interval.

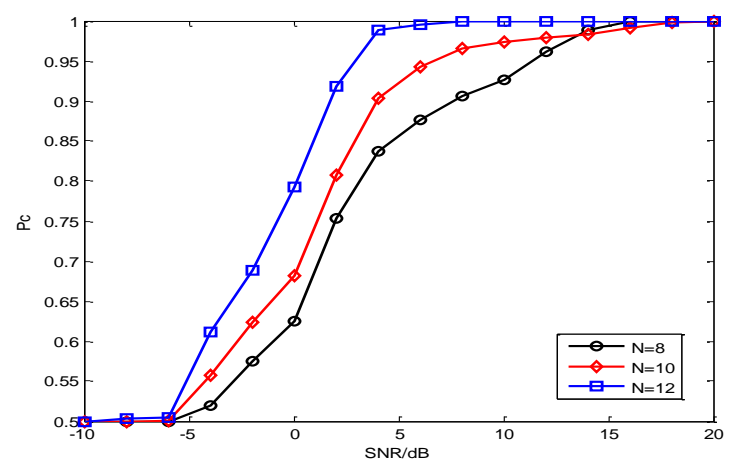

Figure 5. The average recognition probability under different sampling factors.

Figure 4 depicts the probability of correct identification for different values of the confidence interval, $\beta$.As $\beta$ increases, the average probability of correct identification of SM also increases, while AL decreases. According to the formula (14), with the confidence interval $\beta$ increasing, threshold $\lambda$ also increases, which yields a better probability of identification.

Figure 5 shows the average probability of correct identification as a function for different value of decimation factor, such as $\mathrm{N}=8,10,12$. As the decimation factor of signal samples increasing, the number of transmission samples is enlarged. In other words, the elements of $F_{y}(\mathrm{x}) F_{z}(\mathrm{x})$ has enlarged so as to influence the empirical calculated distribution function, which makes a better identification effect.

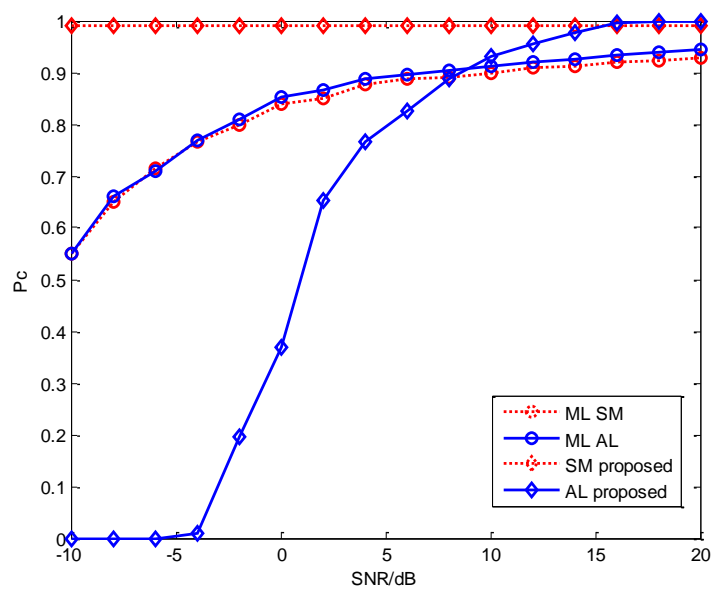

Figure 6. The performance comparison of proposed algorithm and ML algorithm.

Figure 6 places the comparison of the probability of correct identification of SM and AL-STBC between the ML algorithm and the algorithm proposed. In the case of low SNR, the performance of ML algorithm is better than algorithm proposed, but with the increase of SNR, the performance of proposed method is significantly better than ML algorithm. 
On the other hand, ML algorithm needs to know channel information, and its calculation is high and complex. The algorithm proposed which employs a single receive antenna, has a more general applicability in the practical system.

\section{CONCLUSION}

A novel and efficient algorithm is proposed for the frequency-selective fading channels to classify STBC via Kolmogorov-Smirnov (K-S) test when a single antenna is employed at the receiver. The algorithm exploits the discrimination features provided by the empirical cumulative distributions (CDFs) of the received signal. The distance between CDFs is employed relies on the two-sample Kolmogorov-Smirnov test. The simulation shows that the proposed algorithm performs well, and yields an increase in the probability of correct identification as the sampling factor and interval increases. The proposed algorithm does not need estimation of channel, noise statistics and is robust to modulation, even with non-Gaussian noise. Finally, compared with the algorithm referred in [9], the proposed algorithm offers a better identification probability, and is suitable for a practical implementation without a high computational complexity.

\section{REFERENCES}

[1] Choqueuse V, Koffi Yao, Ludovic Collin, et al. Hierarchical space-time block recognition using correlation matrices [J]. IEEE Transaction on Wireless Communication, 2008, 7(9): 3526-3534.
[2] Choqueuse V, Marazin M, Collin L, et al. Blind recognition of linear space-time block codes: a likelihood-based approach [J]. IEEE Transaction on Signal Processing, 2010, 58(3): 1290-1299.

[3] Eldemerdash Y A, Marey M, Dobre O A, et al. Fourth-order statistics for blind classification of spatial multiplexing and Alamouti space-time block codes signals [J]. IEEE Transaction on Communication, 2013, 61(6): 2420-2431.

[4] Marey M, Dobre O A, Inkol Robert. Classification of space-time block codes based on second-order cyclostationarity with transmission impairments [J]. IEEE Transaction on Wireless Communication, 2012, 11(7): 2574-2584.

[5] Y. Eldemerdash, M. Marey, O. Dobre, G. Karagiannidis, and R. Inkol, Fourth-order statistics for blind classification of spatial multiplexing andalamouti space-time block code signals, IEEE Trans. Commun., vol. 61,no. 6, pp. 2420-2431, Jun. 2013.

[6] M. R. De Young, R. Heath, and B. L. Evans, Using higher order cyclostationarity to identify space-time block codes, in Proc. IEEE GLOBE COM, 2008, pp. 1-5.

[7] Choqueuse, K. Yao, L. Collin, and G. Burel, Hierarchical space-time block code recognition using correlation matrices, IEEE Trans. Wireless Common., vol. 7, no. 9, pp. 3526-3534, Sep. 2008.

[8] Mohammad karimi M, Dobre O A. Blind identification of spatial multiplexing and Alamouti space-time block code via Kolmogorov-Smirnov (K-S) test [J]. IEEE Communication Letters, 2014, 18(10): 1711-1714.

[9] Mohamed Marey, Senior Member, Classification of STBC Systems Over Frequency-Selective Channels [J]. DOI10.1109/TVT.2014.2335415, IEEE Transactions on Vehicular Technology.

[10] Yi Sha, Bin Wang. The performance of STBC over frequency-selective fading channels[J] Journal of Northeastern University vol.28, No.8,Aug.2007:1113-1116. 